\title{
CONFORMACIÓN DE LOS ESPACIOS DE PODER EN EL GOBIERNO DE LA UNIVERSIDAD NACIONAL DE CÓRDOBA (ARGENTINA) A MEDIADOS DEL SIGLO XX
}

\author{
Norma Dolores Riquelme * \\ CONICET - Argentina. Grupo de Investigación HISULA. SHELA \\ nriquelme@arnet.com.ar
}

Recepción: 30/05/2008

Evaluación 01/06- 15/09/2008

Aceptación: 29/09/2008

Artículo de Reflexión

\section{RESUMEN}

Este trabajo, reseña la forma en que los alumnos de las universida-des nacionales argentinas tuvieron acceso al gobierno universitario y al poder que esto significa al culminar la década de los años cincuenta. Ello fue la consecuencia de un movimiento iniciado a principios del siglo XX y que por los avatares políticos de la Argentina, no pudo materializarse sino a mediados de dicho siglo. El cogobierno caracterizó las relaciones de poder durante casi todo el tiempo que siguió, aún cuando los gobiernos de facto intentaron eliminarlo, y hoy es parte ineluctable de la vida universitaria, con todo lo que ello conlleva.

Palabras Claves: Política Universitaria, Movimiento Estudiantil, Universidad.

Doctora en Historia, Investigadora independiente de CONICEF. Argentina, miembro del Grupo de Investigación Historia y Prospectiva de la Universidad Latinoamericana HISULA. Ha participado en 50 Congresos de la Especialidad y ha escrito más de 90 trabajos de investigación. 


\title{
ESTABLISHING POWER SPACES IN THE GOVERNMENT OF THE NATIONAL UNIVERSITY OF CORDOBA-ARGENTINA IN THE MIDDLE OF THE $20^{\mathrm{TH}}$ CENTURY
}

\author{
Norma Dolores Riquelme \\ CONICET - Argentina. Grupo de Investigación HISULA. SHELA \\ nriquelme@arnet.com.ar
}

\begin{abstract}
Through this piece of work, it is our intention to report the way in which the students of Argentinian national universities had access to the university government and to the power that it involves- at the end of the 50s. All that was the consequence of a movement which started at the turn of the XX century and which, due to the political vicissitudes of Argentina, could not take place until the mid-twentieth century. The co-government featured the power relationships throughout the time after, even when the coups d' etat attempted to eliminate it. Today, it is an unavoidable part of our university life, with all which that implies.
\end{abstract}

Key Words: University politics, student Movement, University.

\section{INTRODUCCIÓN *}

En 1918 los estudiantes universitarios cordobeses promovieron la Reforma Universitaria que, desde el principio, manifestó ser liberal y progresista y, por ello, aspirar a construir una universidad científica; esto es, contraria al dogmatismo y a las tendencias clericales y reaccionarias que se le endilgan a la Universidad de Córdoba. E inmediatamente, reclamaron su participación, junto a graduados y docentes, en el máximo gobierno de la misma, materializando así un reclamo

\footnotetext{
* Sobre este mismo tema nos hemos explayado con anterioridad en "Los espacios de poder en la universidad argentina de mediados del siglo XX. El caso de Córdoba” en Actas do XII Congreso Internacional de Ahila, volumen IV, editadas por Eugenio Dos Santos, Asociación de Historiadores Latinoamericanistas Europeos (AHILA) y Centro Leonardo Coimbra, Faculdade de Letras da Universidade do Porto, 2002 y también en "El debate acerca de los espacios de poder en la universidad argentina de mediados del siglo XX. El caso de Córdoba”, en Primer Congreso Internacional sobre Historia de las Universidades en América y Europa, Córdoba, 2003.
} 
que, a nivel teórico, fuera planteado diez años antes en el Primer Congreso Americano de Estudiantes.

Los cabecillas de ese movimiento se autotitularon "liberales", englobando en tal palabra todas las libertades y adelantos que el mundo moderno había puesto en sus manos. La democracia estaba en boca de todos y, por entonces, se encontraba muy consustanciada con el voto universal como lo muestra el hecho de que, pocos años atrás, se había consagrado la ley de sufragio universal masculino en la Argentina. ${ }^{1}$ Los jóvenes universitarios fueron testigos de las polémicas que sobre el tema se generaron dentro de la sociedad de su tiempo, por lo que no es de extrañar que se sintieran legítimamente autorizados a reclamar su participación en la elección de las autoridades universitarias; claro está que fueron más allá, al demandar también compartir responsabilidades. Efectivamente, su solicitud se orientaba en dos sentidos: el primero estaba referido a su calidad de electores; el segundo, en cambio, era más amplio y tenía que ver con la condición de "elegibles", en los comicios de autoridades universitarias.

Quizá ello pueda entenderse mejor si se toman en cuenta otros sucesos que sacudían a la Argentina de aquellos días en la búsqueda de lograr una mayor igualdad, tales como los reclamos obreros en demanda de legítimas reivindicaciones y la acción de los partidos políticos en busca de una mayor dignificación del ciudadano; movimientos paralelos a los cambios trascendentes que se planteaban a nivel mundial como consecuencia de la primera gran guerra y la revolución rusa de 1917. Los jóvenes se sintieron protagonistas en una época de cambios y esta idea, aparecida en los preludios del siglo XX, estuvo avalada por gran parte de los intelectuales del país, sobre todo por aquellos que, impactados por las promesas intelectuales que el nuevo siglo ofrecía, se disponían a construir una nueva Argentina.

El reclamo del sector estudiantil no era descabellado, sobre todo en lo que hace al voto activo, pues podría entenderse que a esos jóvenes, preparados y mayores de edad en muchos casos, se los estaba discriminando. Pues, ¿cómo explicar que si estaban capacitados para participar en las elecciones comunes se los excluyera de las elecciones universitarias?. Por otra parte, debe tenerse en cuenta que la juventud universitaria, prácticamente en su totalidad masculina, constituía una élite a la que se preparaba para gobernar, según solía hacerse notar en los discursos de graduación; ¿no sería razonable, entonces, que se

\footnotetext{
${ }^{1}$ El pensamiento occidental a lo largo del siglo XIX entendió que la única forma de democracia compatible con el Estado liberal es decir, con un Estado dispuesto a reconocer la libertad de pensamiento, religión, imprenta, reunión etc. era la democracia representativa, donde la tarea gubernativa quedaba a cargo de un grupo de elegidos por aquellos que la ley capacitaba para votar.
} 
ejercitara en el gobierno de la universidad? Ninguno de los pensadores de la época lo manifestó explícitamente de esta manera; pero ello pareciera desprenderse del tratamiento que recibió el tema.

También contribuye a explicar esta postura el hecho aceptado de que la juventud constituye un dinámico factor de cambio y que sus acciones naturalmente se caracterizan por su energía, vitalidad y entusiasmo. Por ello, permitirle desempeñar tan importante papel, sería la mejor manera de producir transformaciones dentro de la alta casa de estudio, cuyo quietismo pretendían sacudir muchos de aquellos actores sociales a quienes les tocó transitar su vida por entonces.

Así fue como destacados pensadores como Alejandro Korn, José Ingenieros o Marcelino Ugarte, proclamaron con encendidas palabras la necesidad de que los alumnos participaran en el gobierno de la universidad. Para no abundar en los ejemplos veamos sólo lo que decía el primero de ellos:

La Reforma es un proceso dinámico, su propósito es crear un nuevo espiritu universitario, devolver a la universidad consciente de su misión y de su dignidad, el prestigio perdido. Al efecto es imprescindible la intervención de los estudiantes en el gobierno de la universidad. Ellos y solamente ellos representan el ímpetu propulsor, la acción eficiente, capaz de conmover la inercia y evitar el estancamiento. Sin ellos nada se habría hecho. La forma en que han de intervenir es cuestión secundaria; lo importante es que constituyan un poder del cual en adelante no se pueda prescindir. Por conquistar o afirmar estepoder la juventud universitaria en un esfueryo solidario que abarca todo el pais, lleva dos años de gallarda lucha, y de su éxito depende el porvenir de la cultura argentina. $^{2}$

Ya en abril de 1918, en un manifiesto elevado al Ministerio de Instrucción Pública, el Comité Pro-Reforma se preguntaba:

¿No es acaso, la actual constitución del gobierno universitario un anacronismo irritante, una flagrante contradicción con los fundamentos de nuestras instituciones politicas? ¿ $O$ es que el régimen aristocrático, batido en retirada por el principio de la soberanía popular, debe mantenerse con todos sus defectosy peligros, alli en la Universidad...? La función electiva es primordial en todo gobierno; de su amplitud depende la selección de los hombres llamados a ocupar

2 KORN Alejandro (1986): "La Reforma Universitaria” en CIRIA Alberto y SANGUINETI Horacio, La Reforma Universitaria 1918 - 1983, tomo I, Centro Editor de América Latina, Biblioteca Política Argentina, Ediciones Especiales, p. 66. 
posiciones dirigentesy su libre y periódico ejercicio garantiza la capacidad de los llamados a ejercerlas.

En el gobierno de la casa universitaria deben formar parte todos los vinculados a la misma, ya sean docentes, educandos o egresados con título, no directamente bajo la forma de corporaciones estables con facultades legilativas o administrativas, sino por modo indirecto mediante la periódica elección de su primera autoridad... El alumno y el profesional egresado, tienen asimismo interés directo en la eleccion; intereses gremiales los vinculan a la universidad... a unos como a otros no puede serles indiferentes el gobierno universitario y es menester otorgarles la participación que les corresponde... Alprocedimiento de la elección... debe incorporarse la práctica del voto secreto, por cuya adopción abogan razones fundamentales que no escaparán al criterio de los miembros de ese Honorable Consejo. ${ }^{3}$

Los principios de soberanía popular y la reivindicación del voto secreto aquí proclamados se reiteraron en el Manifiesto Liminar, fechado el 21 de junio de ese año, donde los reformistas reclamaron "un gobierno estrictamente democrático" y sostuvieron " que el demos universitario, la soberanía, el derecho a darse el gobierno propio radica principalmente en los estudiantes". ${ }^{4}$ La primera y osada manifestación de la resolución de pasar a la praxis y apropiarse del gobierno universitario se ofreció en los agitados días siguientes, cuando la Federación Universitaria decidió ocupar la Universidad de Córdoba y designar una Junta Ejecutiva, integrada por tres estudiantes, para hacerse cargo del gobierno provisional de la misma y, otros tres, para ejercer los correspondientes decanatos.

Contemporáneamente, los ecos de lo acaecido en la Casa de Trejo sacudieron a la universidad de Buenos Aires que, en octubre de ese mismo año, reglamentó la elección de autoridades para esa Casa con intervención de los alumnos. Esto valió el siguiente comentario del diario La Prensa:

Por primera vez se confía la reorganización universal a la acción verdaderamente democrática, de lo que podríamos llamar el 'pueblo', es decir, los calificados para votar en cada Facultad.

Decimos verdaderamente democrática, porque la democracia requiere electores conscientes, de caráctery nobles intenciones, y en ninguna parte es dado ballar en mayor grado aunadas estas calificaciones del ciudadano, que en las agrupaciones universitarias!.

${ }^{3}$ DEL MAZO, Gabriel, (Compilador). (1941): La Reforma Universitaria, Tomo I: El Movimiento argentino (1918 - 1940), La Plata, Edición del Centro de Estudiantes de Ingeniería, 12 (Memorial de anhelos y exposición de cargos. (El Comité Pro- Reforma Universitaria ante el Ministerio de Instrucción Pública y Consejo Superior de la Universidad)

${ }^{4}$ IDEM, Ibídem, 2. Como es sabido el manifiesto fue redactado por Deodoro Roca, un doctor en derecho egresado pocos años antes y conocido por su inteligencia pero también por sus tendencias transgresoras, las que le depararon tanto el odio de sus opositores como la fascinación y el respeto de sus amigos y seguidores.

${ }^{5}$ Buenos Aires, 4 de octubre de 1918. 
Sin embargo, pasada la euforia que siguió a la Reforma, los gobiernos tendieron a restar influencia a los estudiantes dentro del gobierno de la universidad. Por entonces el país comenzaba un viraje hacia el nacionalismo, arrastrando en pos de sí a muchos antiguos reformistas. Fue así como, en 1923, el presidente Alvear, buscando terminar con la oposición que sentía y presentía en las universidades, decretó la intervención de la del Litoral y, poco después, de la de Córdoba. Esa política fue, con ligeras variantes, continuada por sus sucesores y se extendió por más de tres décadas, en las cuales los gobiernos mantuvieron cercenadas las libertades de las altas casas de estudio. No obstante, la generación del Centenario había colocado en manos de los estudiantes una bandera que no dejarían caer más y, durante ese período, siguieron trabajando incesantemente en pos de lograrlo.

Los gobiernos peronistas significaron un significativo retroceso en ese proceso y volcaron a gran parte de los estudiantes universitarios a una cada vez más activa oposión política, que terminó de plasmarse en su participación en los "comandos civiles" de la Revolución Libertadora de 1955. En parte fue esa participación la que les permitió, una vez caído Juan Domingo Perón, acceder por fin, como recompensa, a lo esperado durante más de treinta años. En 1956, los jóvenes volvieron a demandar la igualdad numérica de docentes, egresados y estudiantes en los máximos órganos de gobierno de la universidad y esta vez lo consiguieron. Aquí, en el fondo, lo que estaba en juego era el reclamo de una importante cuota de poder dentro de una Argentina que procuraba reorganizarse, a veces volviendo a los patrones existentes antes de 1945 y, en otras, introduciendo novedades. La metodología utilizada giró en tormo a la historia político-social de la educación y se sustenta en fuentes primarias.

\section{El poder}

Esta palabra, como ocurre con casi todas, ha variado su sentido con el transcurso de la historia, siendo motivo de sesudas opiniones que numerosos especialistas han vertido sobre esta cuestión. ${ }^{6}$ Muchos coinciden en que, en sentido amplio, la palabra "poder" designa la capacidad de imponer la propia voluntad y puede hacer referencia tanto a un solo individuo como a un grupo; es obvio que, en nuestro caso, nos referimos a la segunda opción. Tiene relación con el concepto de dominación, el cual implica la obediencia a un determinado mandato; ella existe cuando alguien manda eficazmente a otro, es decir cuando

6 Existen muchísimas obras valiosas acerca de este tema, pero todas referidas al mundo político que es, en realidad, lo que preocupa a los teóricos. Nosotros nos hemos limitado a transferir aquellos conceptos que, a nuestro criterio, se adaptan a un caso particular, tal como lo es la universidad. 
éste debe obedecer. En este sentido o sea en relación con la vida del hombre en sociedad, el poder se convierte "en capacidad del hombrepara determinar la conducta del hombre:poder del hombre sobre el hombre". ${ }^{7}$ Efectivamente, está claro que no hay poder si no hay, junto al individuo o grupo que lo ejerce, otro individuo o grupo que se ve inducido a comportarse del modo deseado por el primero y, si no se da esta condición, el poder desaparece. Por lo tanto, él tiene que ver con las relaciones entre los seres humanos. Es también, desde ya, una relación desigual, en tanto la voluntad de algunos prevalece sobre la de los otros. ${ }^{8}$

En el caso que nos ocupa, la situación está amparada por la existencia de una reglamentación que regula tanto los deberes y las funciones de los detentadores como de los receptores del poder. Pero la organización creada al amparo de esa legislación no es estática sino que, por el contrario, los detentadores están facultados, en buena medida, para trasformarla en caso que lo juzguen necesario. Esto es lo que Max Weber denominó una asociación de dominación, en la que las personas quedan sometidas a relaciones de dominación en virtud del orden vigente, el que varía según las circunstancias. ${ }^{9}$ Este mismo autor también definió a esta situación como autoridad legal o dominación legal, o sea aquella dominación racional que se asienta en la existencia de normas pre-establecidas que regulan la actividad y marcan los límites y las atribuciones tanto de los detentadores como de los receptores del poder. La modificación o permanencia de determinados patrones de conducta dependerá de las pautas políticas que se adopten y su aplicación exitosa se sujetará a las estrategias de dominación que se apliquen. Efectivamente, dichas pautas determinan el tipo de dominación que se ejercerá en cada esfera. Ellas pueden perseguir fines académicos, económicos, racionales etc, pero también otros utilitarios o ideológicos; y aún otros más falaces que podrían ocultarse tras la fachada de fines generales. En el caso que nos interesa, la esfera delpoder está limitada al ámbito de la Universidad pero, muchas veces, trasciende ese marco y se convierte en el trampolín de acceso a otras escalas de la esfera pública. En efecto, son muchos los dirigentes argentinos de todo tipo que hicieron sus primeras armas en el ámbito de la política universitaria.

Además, el ejercicio del poder no siempre es unidireccional, es decir que uno manda y el otro obedece, sino que, las más de las veces, las relaciones son recíprocas, es decir que dependen de ciertas negociaciones con otro sector del poder o con grupos electorales. Cada uno usará los medios de que disponga -

7 STOPPINO, Mario. (1995): Poder, en Gianfranco Diccionario de Política, BOBBIO, Norberto, MATTEUCCI Nicola y PASQUINO, México, Siglo Veintiuno Editores, (octava edición en español).

${ }^{8}$ MARIANI, Víctor. (1986): "Introducción al estudio del poder" en Estudios sobre la sociedad y el Estado, ROUDIL, Héctor. (Compilador), Buenos Aires, Eudeba, pp. 231 y ss.

${ }^{9}$ Conf. Economía y sociedad. Esbozo de sociología comprensiva, México, Fondo de Cultura Económica, 1997 (undécima reimpresión), 172 y ss. 
a veces transparentes y otras no tanto- para influir en la conducta de terceros y, a su vez, deberá hacer concesiones. En el caso que nos ocupa, los electores, esto es el total de los estudiantes, no gozan de peso político posterior al momento del ejercicio del voto. Pero sí existen importantes relaciones mutuas entre los distintos estamentos que detentan la autoridad y de todos ellos con los decanos y el rector, quienes deben su nombramiento a las negociaciones realizadas con todos y cada uno de los factores de poder que estamos mencionando.

Los modos de ejercer el poder son muchos y pasan ya por la persuasión, el ruego y aún la manipulación, como por un juego de amenazas, intimidaciones y recompensas, admitiendo, como es fácil darse cuenta, un amplio abanico de otras posibilidades donde no se articularían relaciones de fuerza entre los actores sociales, o sea relaciones causales, sino que estas serían teleológicas, pues las respuestas de parte de los receptores dependerían de modificaciones en su razonamiento. ${ }^{10} \mathrm{Y}$ esta circunstancia se complica si pensamos que se trata de un gobierno colegiado en el que las decisiones pueden adoptarse por unanimidad o por mayoría. La colegiatura, en el caso de la universidad, descansa en la conducción de la misma mediante lo que Weber denominó — siempre en referencia al mundo político — un colegio de partidos. Pone freno a las decisiones personales y rápidas del rector y los decanos e impone, en cambio, las aspiraciones del partido mayoritario representado en los Consejos.

Numerosos especialistas han supuesto que teniendo claro en qué consiste el poder, se puede medirlo; no obstante muchos otros estiman que hablar de poder es hablar de una noción filosófica y por lo tanto no cuantificable científicamente. ${ }^{11}$ Además existe acuerdo acerca de la presencia de un poder oculto, "no observable" ¿Cómo medirlo entonces?. No obstante, existen propuestas sobre cómo cuantificar distintas opciones relacionadas con el poder las que, hasta hoy, no gozan de la común aceptación de todos los especialistas.

En sentido amplio importa también tener en cuenta a cuántas personas afecta. En este caso, el ambiente universitario cordobés compromete a un diez por ciento de la población total de la ciudad donde se ubica la universidad y, dentro de esa esfera, el sector de los "no docentes", como normalmente se denomina al personal administrativo, está menos sujeto que el resto a la voluntad

${ }^{10}$ LAPORTA Francisco, "Poder y derecho” en El derecho y la justicia, GARZÓN VALDEZ Ernesto y LAPORTA Francisco (Ed.), Madrid, Consejo Superior de Investigaciones Científicas, Boletín Oficial del Estado, Editorial Trotta, 1996, 441 y ss. No nos extendemos en los interesantes aportes de este autor, debido a las restricciones de espacio.

${ }^{11}$ El norteamericano Wright Mills avanzó en este sentido; no obstante otros investigadores entendieron que su postura no era científica. Conf. La élite del poder, México - Buenos Aires, Fondo de Cultura Económica, 1957. 
del Consejo Superior y los Directivos y está resguardado, en cambio, por conquistas gremiales. Pero tampoco debe ignorarse la influencia que la universidad tiene en la sociedad en general, lo cual inviste a sus autoridades de un "poder adicional" dentro de la esfera política que la circunda.

Somos conscientes del esquematismo que necesariamente tiene un esfuerzo de síntesis sobre un tema tan complejo. Pero entendemos que es necesario intentarlo, puesto que los teóricos afirman que el poder constituye una variable fundamental en todos los sectores de análisis de la política. Ello es válido también para el estudio de las organizaciones, en nuestro caso la universidad, donde la estructura jerárquica y las formas que ella asume ponen naturalmente en primer plano el fenómeno que nos ocupa.

En el apartado que sigue intentaremos reseñar cómo jugaron en la práctica las variables que llevaron al estudiantado de Córdoba a conseguir una representación verdaderamente significativa en el gobierno de la Universidad, teniendo presente que ellos y los docentes que los acompañaban sabían que del sector político que logre imponerse en tan alto sitial, dependería la política que seguirían en el futuro las altas casas de estudio.

De todas maneras, nos apresuramos a reconocer que es muy difícil por el momento desentrañar el exacto alcance del poder alcanzado por el estamento estudiantil dentro del ámbito de la Universidad -tema que, como tantos otros referidos a las altas casas de estudio, aún espera el interés de los especialistas-; y menos poder explicitarlo en una comunicación de pocas páginas.

\section{El acceso al poder}

La década de los años cuarenta y mitad de los cincuenta estuvo dominada en la Argentina por el peronismo caracterizado, sobre todo en el último tramo, por fuertes matices autoritarios y por la presencia de una oposición cada vez más combativa. De todas maneras, a partir de 1947, los alumnos ya habían obtenido por primera vez un espacio legal de participación en el gobierno universitario, cuando la ley 13.031 permitió la inclusión de uno de ellos en el Consejo Superior con voz pero sin voto; pero, en la práctica, su intervención había quedado reservada a los leales al régimen. A partir de entonces los jóvenes ya no abandonarían el protagonismo adquirido que se iría consolidando, 
precisamente, en las duras luchas contra el gobierno de Perón. ${ }^{12}$ Efectivamente, gran parte de los estudiantes universitarios enfrentaron al régimen desde el primer día y jugaron un papel importante en su debilitamiento. Como contrapartida, el peronismo fue francamente restrictivo respecto a la universidad. La Constitución reformada de 1949, introdujo algunas variantes respecto a lo que ya se había dispuesto en la ley universitaria $\mathrm{N}^{\mathrm{0}} 13031$.

La nueva norma legal fijaba como objetivo de las universidades la enseñanza en el grado superior, la formación de los jóvenes para la vida, el cultivo de las ciencias y el ejercicio de las profesiones liberales, siempre subrayando su sentido social, eje sobre el que el peronismo hacia girar todo su gobierno. El artículo $4^{\circ}$, referido a las funciones específicas, prohibía la actividad política partidista en las universidades y, al respecto, el propio Perón decía que en las universidades se debía trabajar para mayor honra del país y para felicidad de todos los argentinos y no para un partido o un grupo. Pero se llegó más lejos aún, cuando la nueva constitución dispuso el establecimiento en las universidades de cursos de formación política, obligatorios y comunes, para los estudiantes de todas las facultades. El gobierno peronista estaba decidido a uniformar el pensamiento de todos los argentinos, y entre ellos el de los universitarios, que intentaron ser adoctrinados de diversas maneras, desconociendo que los jóvenes son poco proclives a aceptar este tipo de adoctrinamiento. El Segundo Plan Quinquenal terminó de sellar lo que el gobierno pretendía en este campo, pero ya era tarde... ${ }^{13}$

${ }^{12}$ La ley 13.031, promulgada el 9 de octubre de 1947, se ocupó de organizar la vida universitaria, otorgándole, al menos en la letra, "autonomía técnica, docente y científica”. El Consejo Universitario se integraba con el rector, designado por el P.E., y por los decanos y vicedecanos. Los estudiantes tenían representación en los consejos directivos por intermedio de un delegado de cada Escuela. El artículo 85 decía: "Entre los diez alumnos que hubiesen obtenido las más altas calificaciones en el transcurso de su carrera y se encuentren cursando el último año, se sorteará el que ha de tener la representación estudiantil. Este cargo es irrenunciable...”. El artículo siguiente rezaba “... el delegado podrá expresar libremente el anhelo de sus representados, no teniendo voto en las decisiones que adopte el Consejo...” Jurisprudencia Argentina, IV, Buenos Aires, 1957, 21. También Anales de Legislación Argentina, Buenos Aires, La Ley, 1947, T. VII, 369 y ss. Por otro lado, de acuerdo al artículo 49 de la ley que estamos recordando se creó, durante los años del peronismo, una agrupación oficialista llamada la CGU (Confederación General Universitaria), que era la única reconocida por el gobierno y posteriormente sólo los afiliados a ella podían ser electos consejeros. Estas leyes se derogaron después de la caída de Perón.

${ }^{13}$ Lo que enunciaba el Segundo Plan Quinquenal fue especificado por la ley 14297/54 que sustituyó a la ley 13031/47. Esta norma determinó un sistema de mayor control por parte del Estado sobre las universidades, destinado a orientar la enseñanza universitaria de acuerdo con los objetivos de la doctrina justicialista. Mediante este instrumento legal, la universidad -ahora centralizada, controlada y sin autonomía real aunque se dijera lo contrario-, quedó adscripta a los propósitos políticos. Según el título V, art. 59 de la misma se establecía que en los consejos de cada facultad habría un representante estudiantil, elegido entre los alumnos que cursaran los tres últimos años de la carrera y "proveniente de entidad gremial reconocida", que tendría voto sólo en las cuestiones que le atañeran directamente. A estos temas se ha referido también DELIA TERESITA ALVAREZ DE TOMASSONE, UNIVERSIDAD OBRERA NACIONAL UNIVERSIDAD TECNOLÓGICA NACIONAL. La génesis de una Universidad (1948 - 1962), Editorial de la Universidad Tecnológica Nacional, 2006. 
Después de la caída del peronismo, en 1955, se produjo una reacción tendiente a proclamar una mayor libertad en casi todos los campos y la universidad no podía quedar excluida. La reorganización de la de Córdoba pasó por la cesantía de quienes habían ocupado cargos durante la gestión peronista y por la reunión del primer Consejo Universitario integrado por un rector con el carácter de interventor y los seis decanos de las respectivas facultades. El gobierno de la revolución intentó recomponer los estudios superiores para lo que dictó el decreto 6.403 que determinó, entre otras cosas, la forma en que se llegaría a la plena autonomía universitaria y la necesidad de que las altas casas de estudio dictasen su propio estatuto una vez que se hubieran constituido los consejos directivos. ${ }^{14}$

Con estos antecedentes el Consejo Superior enfrentó, en los meses siguientes, los preparativos para la redacción del Estatuto Universitario el que, de acuerdo al decreto 10.775 , debía ser encarado por docentes, egresados y estudiantes. ${ }^{15}$ El mismo, para entrar en vigencia, debería ser aprobado por los consejos directivos y también por la Asamblea Universitaria a donde concurrirían

${ }^{14}$ Este decreto ley se justificó en la necesidad de recuperar las universidades, fundándose en el principio de la autonomía "cuyo valor y eficacia ha proclamado reconociéndolo como el fundamento de la responsabilidad que incumbe a dichas organizaciones para darse a sí mismas su estructura y funcionamiento de acuerdo a las finalidades que le son propias”. El decreto, en su artículo 3, establecía que los consejos directivos se integraban con representantes de los profesores titulares, adjuntos, egresados y estudiantes en el número que determinase cada universidad según sus particulares modalidades y conveniencias "y en una proporción que asegure la responsabilidad directiva de los representantes del claustro de profesores". Los alumnos que actuasen como representantes debían haber aprobado las tres cuartas partes de la carrera. El Consejo Superior se integraba con los decanos, alumnos y egresados y estaba presidido por el rector. Como en el caso anterior se recomendaba que el número debía establecerse "en una proporción que asegure la responsabilidad directiva de los representantes del claustro de profesores”. La autarquía quedaría plenamente otorgada después que se integraran los cargos de profesores por concurso y que los interventores los llamaran a elecciones junto con los alumnos y egresados para constituir los consejos. Jurisprudencia Argentina, 1956, I, Buenos Aires, 51 y ss. A criterio de Avelino José Porto esta ley, como otras, fue la expresión de una posición político-ideológica más que de un orden científico -académico. Conf. "Las Leyes, la Universidad y el País” en Todo es Historia, n 147, agosto de 1979, 24. Sobre la autarquía decía uno de los miembros del Consejo Superior "La autarquía lleva implícitos tres conceptos: Facultad del gobierno propio en lo atinente a lo administrativo, personalidad jurídica y patrimonio propio, todo lo que está contemplado en el decreto ley 7361 y que ratifican las consideraciones en que se funda el 6403... El concepto de autarquía no debe confundirse con el concepto de vida descentralizada a que se refiere el decreto en que esta última interpretación se funda, pues las universidades todavía no tienen la amplia libertad que desde un primer momento se ha querido darle y que todos anhelamos".

15 Esta ley creó el Consejo de las Universidades Nacionales, dentro del programa general de "fortalecer la descentralización administrativa, estableciendo las condiciones jurídicas, económicas y culturales que propendan a ellos como límites al poder central" y para "suprimir todos los vestigios de totalitarismo" y "restablecer el imperio de la... libertad y de la democracia”. El gobierno estimaba que había llegado el momento de proclamar la autonomía universitaria para lo cual había que dictar la ley que autorizara a cada universidad a dictar su propio estatuto. Para ello se creaba el Consejo 
representantes de los tres estamentos. Era la primera vez que los estudiantes tendrían voz y voto y era esta una experiencia sin precedentes en el mundo que fue encarada pensando que esa sería la solución "argentina" para problemas "argentinos". 16

Mucho podríamos decir sobre este tema; no obstante lo que parece más digno de destacar es la firmeza y entusiasmo con que este grupo encaró el tema, la intención de evitar imitaciones y el convencimiento de la necesidad de obedecer a un modelo argentino, a pesar de las diferencias ideológicas que separaban a varios de los decanos. ${ }^{17}$

Una vez vencido el peronismo, los estudiantes se reorganizaron resurgiendo la Federación de Estudiantes Universitarios y la Federación de Agrupaciones Universitarias Integralistas, logrando ambas el mayor consenso entre los jóvenes. A su lado trabajaron, también, el Ateneo Universitario, la Liga Humanista y el Centro Superación de Estudiantes de Farmacia y Bioquímica. ${ }^{18}$ En los primeros meses del año 1956, las

de la Universidad que lo haría de acuerdo a la tradición y la conveniencia de cada universidad, pero, como en el caso anterior, se recomendaba "establecer para la constitución y composición de las autoridades de cada una de las Facultades y de la Universidad misma, la proporción que asegure la responsabilidad directiva del claustro de profesores". Jurisprudencia Argentina, 1956, III, Buenos Aires, 76 y ss. ARCHIVO DE LA UNIVERSIDAD NACIONAL DE CÓRDOBA (en lo sucesivo AUNC), Actas del Honorable Consejo Universitario, tomo I, (14 de junio al 15 de noviembre de 1956), fl. 215 y 216.

${ }^{16}$ En Europa sólo los gremios de estudiantes italianos habían comenzado a reclamar participación en el gobierno universitario. En América se había aplicado un sistema similar en una universidad privada de Bolivia y, como forma experimental, se aplicó en México siendo luego suprimido. AUNC, Actas de la Asamblea Universitaria, 1957 1958, tomo I, folios 167 r. y 169 v.

${ }^{17}$ El decano de la Facultad de Derecho fue encargado por sus pares de buscar antecedentes dentro y fuera del país "con el objeto de tener una visión completa de lo que debe ser una universidad autónoma y democrática" y se pensó, también, en traer a Córdoba delegados de otras universidades con más experiencia en este tema. Sin embargo, la tendencia general propendía a que, de acuerdo al decreto, el Estatuto debía acordar con las características y modalidades locales. Conf. AUNC, Actas del Honorable Consejo..., op. cit, fl. 71 a.

18 "Aunque cada uno de los grupos de izquierda tenía su expresión universitaria, ella no se definía abiertamente como brazo político de un partido. $Y$ los comunistas-que protagonizaron en competencia con los radicales los primeros años de este períododefendieron un "movimiento reformista" que se expresaba con diferentes nombres y diferentes siglas en cada una de las facultades. Esta "identidad reformista" compitió, desde fines de los cincuenta, con las diferentes líneas del humanismo cristiano que prevalecieron primero en la derecha para pasar luego muy rápidamente a la franja "progresista": el enfrentamiento humanismo/reformismo, en sus episodios fundamentales, se ubicó del centro a la izquierda del espectro ideológico -y los integrismos y humanismos de derecha, victoriosos en muchas universidades del interior, se corrieron en el curso de muy pocos años, hacia la zona radicalizada del pensamiento cristiano." Beatriz Sarlo, "Estudio preliminar" en La batalla de las ideas (1943-1973), Biblioteca del Pensamiento Argentino, VII, Buenos Aires, Ariel, 2001, 68 . 
agrupaciones estudiantiles se lanzaron a una amplia campaña política que contó con el apoyo y la difusión de los medios de prensa, contagiados por el entusiasmo juvenil. Es necesario tener en cuenta que estas organizaciones eran una extensión de los sectores políticos vigentes y que en su dirigencia, como es lógico, estuvieron los jóvenes más politizados, mientras la mayoría colaboró circunstancialmente o, simplemente, con su voto. ${ }^{19}$

Para entonces las agrupaciones estudiantiles estaban lanzadas a una activa campaña tendiente a consagrar definitivamente una mayor inserción de los estudiantes en el gobierno universitario. Parte de ella fueron distintas manifestaciones y el envío de notas al rector de la Casa de Trejo, que motivaron su reacción y el inicio de una controversia que dificultaría el entendimiento posterior. ${ }^{20}$ No obstante, mientras los enfrentamientos continuaban y las manifestaciones cubrían las calles de la ciudad sede de la universidad más antigua del país, las agrupaciones estudiantiles, y en especial la Federación Universitaria, avanzaban en el análisis de la futura política universitaria. ${ }^{21}$ Ellos estaban convencidos que la intervención estudiantil era el único camino viable para revertir la declinación de las universidades, sobre cuyo deterioro existía un consenso generalizado. ${ }^{22}$

${ }^{19}$ Durante los últimos años de la década de los '60 y los primeros de la del '70 las agrupaciones estudiantiles tuvieron un altísimo grado de politización. Unas se inclinaron a la ultra derecha, otras a la ultra izquierda, mientras algunas, sin irse a los extremos, buscaron su identidad en algunos partidos mayoritarios. Un ejemplo de amplia participación de los jóvenes de Córdoba en las luchas políticas y sociales de la época, se tuvo en el conocido como Cordobazo, producido el 29 de mayo de 1969. La incidencia de estas agrupaciones en la sociedad toda y en la vida universitaria sirvió de pretexto a distintos gobiernos militares para prohibir su actividad.

${ }^{20}$ La máxima autoridad universitaria, quizá eludiendo su propia responsabilidad, incriminó a los estudiantes la necesidad de buscar el armónico desarrollo de sus actividades dentro del marco de la democracia, "porque el ejercicio de la libertad implica también enorme responsabilidad para la juventud" AUNC, Actas del Honorable Consejo Universitario, 1956, op. cit., fl. 100 v.

${ }^{21}$ La Federación Universitaria se reunió en el verano de 1958, período normal de receso, para organizar una convención destinada a estudiar las bases para la Ley y el Estatuto Universitario. Ella se convocó para el siguiente mes de marzo, teniendo en cuenta que se esperaba que el Congreso Nacional pronto consideraría tal cuestión y se invitó a 4 profesores, 4 estudiantes y 4 graduados de cada universidad. AUNC, Actas del Honorable Consejo Superior, tomo I (24 de enero al 25 de abril de 1958), fl. $118 \mathrm{v}$. ${ }^{22}$ Por aquellos años los estudiantes afirmaban que la educación era "vergonzosa", concepto que sin ser tan frontal, compartían los docentes, quienes aceptaban que ella no estaba de acuerdo con el prestigio de la Casa de Trejo. Se afirmaba que esta situación era el corolario “de lo que pasó en los últimos años” y el propio rector aseguraba que se había entrado "en un peligroso descenso de la autoridad y prestigio de nuestra universidad" y que, en otras partes del mundo ya se sabía que en la Argentina, y particularmente en Córdoba, podía obtenerse un título con un mínimo de esfuerzo. AUNC, Actas de la Asamblea..., tomo I, op. cit., fl 143 r. En los años que siguieron tampoco se acalló la polémica suscitada por el cogobierno y esto puede verse, por ejemplo, en el párrafo que adjuntamos: “Así pasa con los enemigos de la Reforma. Por lo pronto la Reforma abre los cauces de la participación, y este es uno de los temas que la antireforma ha retomado para restablecer la tesis de la ingobernabilidad por 
El gobierno provisional que regía al país por entonces, consciente de la fuerza del estudiantado puesta de manifiesto en su lucha contra el gobierno de Perón y cuyo peso político había crecido notablemente después de su caída, no tenía intenciones de repetir la historia y creyó apaciguarlo tanto con una amnistía, que comprendió a aquellos que habían intervenido en los hechos de violencia acaecidos durante ese mes de mayo, dictada en octubre de 1956; ${ }^{23}$ como con los decretos 6.403 y 10.775 que hemos mencionado anteriormente y que tendían a reglamentar la autonomía universitaria.

Pero el estudiantado cuestionó severamente el alcance de los mismos entendiendo que ellos cercenaban el derecho de los estudiantes al "asegurar la responsabilidad directiva de los representantes del claustro de profesores" y, consecuentes con este pensamiento, la Federación Universitaria se negó a colaborar en la redacción del Estatuto Universitario, previsto en los decretos leyes de la Revolución Libertadora.

No obstante, el 30 de abril de 1957, el Consejo Superior decidió tratar el proyecto del Estatuto que, luego, sería sometido a la Asamblea Universitaria. Los decanos allí presentes convinieron en la necesidad de aprobarlo como un paso trascendente en la conformación de la universidad que todos anhelaban. ${ }^{24}$ No obstante, el de la Facultad de Derecho y Ciencias Sociales creyó necesario explicar que el proyecto no aspiraba a ser un instrumento perfecto y definitivo, sino que solo representaba un paso adelante hacia la universidad que él, como reformista, ambicionaba. Y señaló, a continuación, las reservas que le merecía el proyecto. Entre otras cosas, afirmó que el gobierno de la universidad debía ser ejercido por el demos universitario, estableciéndose la igualdad de representación para profesores, estudiantesy graduados... y que los estudiantes debían estar representados,

medio de los mecanismos establecidos gracias a la Reforma... La Reforma, al abrir los cauces de la participación, estableció basándose en la autonomía una práctica de autogobierno llamado cogobierno, que permitió ensanchar cauces que hicieran parte... todos los sectores que de una u otra manera conformaban la vida y el destino universitario. $Y$ hoy sus críticos señalan que esa participación puede llevar a la ingobernabilidad del sistema.” VANOSSI, Reinaldo. (1989): “Balance de los setenta años de la Reforma Universitaria” en La Reforma Universitaria 1918 - 1988, Buenos Aires, Ministerio de Educación y Justicia de la Nación, Universidad Nacional de Córdoba y Fundación Friedrich Ebert, 189 y ss.

${ }^{23}$ En los considerandos del decreto se decía que "ciertas discrepancias circunstanciales referidas a medios y procedimientos para alcanzar aquellos fines han desembocado en situaciones de tensión y aún de violencia, sólo explicables por la impaciencia y el apasionamiento puestos en la defensa de una causa noble en sí misma”. Conf. Anales de Legislación Argentina, 1956, tomo XVI A, Buenos Aires, La Ley 1048.

${ }^{24}$ El rector interventor era Jorge Núñez. Los decanos presentes eran Santiago Monserrat (de la Facultad de Derecho y Ciencias Sociales), Florencio Ponce (de Odontología), Tomás de Villafañe Lastra (de Medicina), Tomás Fulgueira (de Filosofía y Humanidades), Carlos Revol (de Ciencias Exactas, Físicas y Naturales), Jaime Roca (de Arquitectura y Urbanismo) y Carlos Pardo (de Ciencias Económicas). Este último falleció poco después. 
en su vida gremial, por un solo Centro, aunque en el seno de la masa estudiantil actuasen diversas agrupaciones. ${ }^{25}$

A pesar de todo, el Estatuto a que nos estamos refiriendo — que debió ser previamente aprobado por el Consejo Superior y que debería serlo por la Asamblea Universitaria- dispuso conformar los consejos directivos con 14 miembros: 5 representantes de los titulares, 3 de los adjuntos y asociados, 4 de los estudiantes y 2 de los graduados. ${ }^{26} \mathrm{El}$ Consejo Superior, por su parte, se integraba con el rector y el vicerrector, que duraban cuatro años en sus funciones, con los decanos, 4 estudiantes y 2 egresados.

En los meses que siguieron se reunieron los consejos directivos y, finalmente, en octubre de 1957, la Asamblea Universitaria. ${ }^{27}$ Esta sería el ámbito en el que los estudiantes, en el marco de su lucha por el poder, plantearían las reivindicaciones que reclamaban como justas. Fue allí que, por intermedio del estudiante reformista Enrique Olivares, la Federación Universitaria justificó el repudio a los decretos 6.403, 10.775 y 8.780 , por "regresivos" y "reaccionarios" y por haberse dictado "a espaldas del estudiantado... lesionando las autonomías de las universidades". Luego sostuvo que se les impone "una determinada proporción que asegure la responsabilidad directiva del claustro de profesores", por lo que se les "ha dado representación estudiantil en una forma mezquina con vozy voto, pero inofensiva por su exigüidad numérica". ${ }^{28} \mathrm{Y}$ basados en esta afirmación los reformistas, que eran mayoría, se negaron a participar en la elección de rector. Los integralistas, por su parte, enfrentaron la posición de sus compañeros entendiendo que en la Universidad existía un orden jerárquico — en este caso los profesores_- que era necesario respetar. ${ }^{29}$

${ }^{25}$ AUNC, Actas del Honorable Consejo..., tomo I, op. cit. fl 116 y 117.

${ }^{26}$ Ese año el Consejo Universitario había fijado las condiciones que se requerían para ser profesor: morales y de capacidad, tanto para entrar como para mantenerse en el cargo; y la periodicidad, destinada a estimularlo en una permanente vigilia. Se había autorizado la existencia de cátedras paralelas y libres. “...tenemos a nuestro frente a un profesor, que si ejerce y cumple con las disposiciones de los Estatutos, será un modelo de profesor... indudablemente, el...que va ha afrontar esta lucha, que va ha representar la carrera docente como la hemos reglamentado, es un sujeto que está dispuesto a grandes sacrificios, porque tampoco... la parte económica puede representar... un estímulo.” AUNC, Actas de la Asamblea..., tomo I, op. cit., fl $165 \mathrm{v}$. A los representantes alumnos, por su parte, sólo se les exigía tener una materia aprobada.

${ }^{27}$ Se reunieron en ella 56 docentes, 26 representantes estudiantiles y 14 egresados. Sin el voto estudiantil se acabó proclamando rector a Pedro León.

${ }^{28}$ AUNC, Actas de la Asamblea..., tomo I, op. cit., fl. 7 r.

${ }^{29}$ Participaron de la Asamblea 7 estudiantes integralistas, en cuyo nombre habló José María Willington. AUNC, Actas de la Asamblea..., tomo I, op. cit., fl. 8v. Cabe recordar que los reformistas habían resultado triunfantes en todas las Facultades con un total de 4.600 votos, mientras las demás agrupaciones habían obtenido 3.600. 
Cuando al culminar el año 1957 la Asamblea comenzó a tratar lo referente al gobierno universitario y la conflictiva representatividad de cada claustro, los asambleístas se fraccionaron en tres grupos: uno —el de la mayoría — aconsejaba mantener los 14 representantes que ya había incorporado el Consejo; otro, prefería 18 en paridad de condiciones: 6 profesores, 6 egresados y 6 alumnos y, el tercero, proponía 11: 7 titulares y 4 adjuntos y que los alumnos tuviesen voz pero no voto. No obstante, los que propiciaban una representación igualitaria pronto adhirieron a la primera propuesta, con lo que quedaron solo dos posiciones.

No escapaba a los que protagonizaron estas jornadas que estaban viviendo una etapa experimental, por lo tanto ninguno tenía claro cuál era el gobierno ideal. No obstante, la mayoría aceptaba la representación estudiantil con voz y voto, entendiendo que ella aportaría nuevos puntos de vista sobre viejas cuestiones, ${ }^{30} \mathrm{y}$ que el propio sistema moderaría las aspiraciones y exigencias de los jóvenes. ${ }^{31}$ Estos aportarían su idealismo "que en algunos casos los lleva a jugarse... sin cálculo y con prescindencia de sus consecuencias"; el entusiasmo, necesario para las grandes empresas, y aún el sentimiento. No seles escapaba, sin embargo, que muchas veces los estudiantes se guiaban por el cálculo y que, seguramente, algunos propiciarían soluciones acordes con sus conveniencias circunstanciales; pero, decían, esto "con la práctica de la representación ha de irse puliendo y ha de irse imponiendo el buen criterio". ${ }^{32}$

Todo esto no era óbice para que se reconociera la necesidad de que dicha representación fuese "discretay en minoría respecto del total que estamos propiciando". 33

\footnotetext{
30 “....me pongo, en este momento, en la suposición de que los profesores encargados de esa función directiva, sean los más capacitados, y me pongo también en la suposición de que los representantes de los estudiantes estuvieran en condiciones de muy poca experiencia. Asimismo, aún en esa suposición, el punto de vista estudiantil nos es necesario para conocer en su integridad esto que es la cosa universitaria: el gobierno de la universidad; aunque más no sea conocer cuáles son sus preferencias, sus aspiraciones, cuáles son los inconvenientes que encuentran en su desenvolvimiento, y también, desde el punto de vista de ellos, cuáles son los remedios que proponen para su solución.” AUNC, Actas de la Asamblea..., tomo I, op. cit., fl 160 v. (Palabras de Carlos A. Tagle).

31 "digo moderen, porque esa representación con voto traerá una mayor responsabilidad al plantear y votar las medidas que ellos propongan, tendrán en cuenta que en el futuro, se habrá de recordar que en parte han sido debidas a su intervención, y esto traerá mayor dominio, un cierto principio de contención en sus planteamientos.” AUNC, Actas de la Asamblea..., op. cit., fl 160 v. (Palabras de Carlos A. Tagle).

32 AUNC, Actas de la Asamblea..., tomo I, op. cit., fl 160 v. y 161 r. (Palabras de Carlos A. Tagle).

33 “...el interés superior de la enseñanza universitaria aconseja mantener esa discreta preeminencia, esta relativa mayoría de representantes de los profesores en el gobierno de la Alta Casa de Estudios. Téngase en cuenta que con la representación paritaria, la... de los estudiantes, unida a una cierta parte de los graduados,... pueden, en momentos delicados, formar una mayoría por sobre los profesores, a quienes no se les puede negar que han llegado a una cierta situación en que se los debe suponer capacitados para el gobierno de esta institución” AUNC, Actas de la Asamblea..., tomo I, op. cit., fls. 160 r. y 161 r y v. (Palabras de Carlos A. Tagle).
} 
Un argumento esgrimido en pro de tal postura era reconocer que los discípulos de hoy, tarde o temprano, serían los profesores; el sistema les garantizaba que, en el porvenir, ellos mantendrían una superioridad moderada sobre los estudiantes del futuro. De lo contrario, llegados a docentes, tendrían que descender frente a sus educandos "para darles a ellos en el futuro esa soberanía que boy se gestionay se desea" ${ }^{34}$ Por otra parte, éstos invocaban la existencia de un orden que no se podía transgredir impunemente: el trabajo, el esfuerzo y el estudio, afirmaban, traen mayor ciencia y habilitan en mayor grado para el ejercicio de las funciones directivas. Esa ciencia — agregaba el informante de la mayoría — era útil al gobierno de la universidad y, en definitiva, también a los bien entendidos intereses de los estudiantes. Y fundamentalmente, argumentaban, era necesario reconocer que los profesores, por el solo hecho de serlo, tenían mayor experiencia.

No obstante, los defensores de los intereses estudiantiles contrarrestaron los argumentos anteriores aduciendo que la mejor preparación científica o docente no implicaba una mayor capacitación para gobernar. ${ }^{35}$ Esta era una función eminentemente técnica que podía "ser desempeñada tanto por los profesores, como por los egresados, como por los estudiantes".

El proyecto de la minoría, que defendía el gobierno exclusivo de los profesores, basó sus argumentaciones en que la Universidad era una comunidad homogénea, porque todas sus partes concurren a un solo fin y, como tal, no admitúa subdivisión en clases y estamentos. Por otra parte, afirmaban, el gobierno de la universidad era predominantemente técnico y no político. Y se preguntaban ¿Qué se pretendió lograr con la autonomía sino precisamente desvincularla del ambiente político?. ${ }^{36}$

Los reformistas eran concientes de la politización de la universidad, pero creían que ella era benéfica y así lo pondría de manifiesto uno de sus principales referentes cuando decía:

... La universidad argentina, como institución... es expresión de la mentalidad oficial. No puede concebirse... una universidad argentina que no sufriera el impacto de dirección y de formación del pensamiento politico dominante, ain cuando el pensamiento politico fuera bandera de un partido o de otro...

${ }^{34}$ AUNC, Actas de la Asamblea..., tomo I, op. cit., fl. 161 v. (Palabras de Carlos A. Tagle). 35 "Hay... excelentes profesores, con grandes conocimientos, sobre los cuales no se puede en absoluto poner en duda su capacidad... pero que desconocen la realidad del gobierno de la Universidad, y no se han preocupado por los problemas de este tipo..." AUNC, Actas de la Asamblea..., tomo I, op. cit., fls. 168 r y v. (Palabras de Jesús Osorio Sánchez).

36 “...la autonomía universitaria es la despolitización de la universidad, y la politización es transformar los organismos directivos de la universidad con el mismo carácter, es hacerle perder a este organismo su significación, su naturaleza predominantemente técnica.” AUNC, Actas de la Asamblea..., tomo I, op. cit., fl. 164 r. (Palabras de Enrique Martínez Paz). 
aún cuando... venimos a enseñary aprender, no conseguimos despojarnos en la puerta de la universidad de lo que politica o socialmente representamos... De que en esta universidad argentina, que debe servir a la sociedad argentina, que no está definida, que está influida por el choque inevitable e ineludible de intereses políticos, económicosy sociales, yo creo que es útil, que es indispensable, absolutamente indispensable, que la universidad se estructure.... con los ingredientes con que está estructurado el pensamiento argentino. ${ }^{37}$

Dicho de otra manera, tampoco se aceptó la propuesta de la minoría de tender a una universidad despolitizada, pues esto colocaría a la universidad en el caso de no poder "ejercer una influencia real sobre los destinos del mundo". ${ }^{38}$ Pero la base de sustentación de la minoría se fundamentó en la idoneidad, principio consagrado por la Constitución como necesario para ser admitido en los empleos. ${ }^{39} \mathrm{Y}$ este fue el tema que, con éxito, lograron desbaratar los representantes estudiantiles. Ellos insistieron en que la idoneidad de los profesores se refería "exclusivamente" a su especialidad y que, en todo caso, su dedicación a la ciencia y al estudio los sacaba de la realidad, en la cual los estudiantes estaban más insertos que los catedráticos. Reconocían su falta de idoneidad, pero decían no entender por qué se hacía hincapié en ella. ${ }^{40}$ En cambio creían en su capacidad para, junto a los profesores, trabajar por una universidad mejor. ${ }^{41}$

No faltó, por supuesto, la palabra de prestigiosos docentes que avalaron la posición estudiantil. ${ }^{42} \mathrm{Ni}$ tampoco quien recordara que en un país que acababa

\footnotetext{
${ }^{37}$ AUNC, Actas de la Asamblea..., tomo I, op. cit., fl. 170 v. (Palabras de Jorge Orgaz).

38 AUNC, Actas de la Asamblea..., tomo I, op. cit., fl. 173 v. (Palabras de Adelmo Montenegro)

39 AUNC, Actas de la Asamblea..., tomo I, op. cit., fl. 165 r. (Palabras de Enrique Martínez Paz)

${ }^{40}$ AUNC, Actas de la Asamblea..., tomo I, op. cit., fl. 169 r. (Palabras del alumno Luis E. Viera Alonso).

41 "Esa es la universidad... con la que sueño que no es esta universidad. Y en esa universidad... no veo porqué el gobierno... debe estar relegado a los profesores cuando ellos solos no forman la universidad. La universidad es la lucha común, el trabajo paralelo, el estar juntos. Eso es Universidad.” AUNC, Actas de la Asamblea..., tomo I, op. cit., fl. 169 v. (Palabras del alumno Luis E. Viera Alonso).

42 “...el concepto de idoneidad adquiere un sentido muy distinto, porque el acto... educativo se agota en la relación recíproca del educando y el educador... es indudable que el criterio de la idoneidad debe ser ampliado y debe abarcar a todos los miembros de la acción educativa. En ese sentido los estudiantes tienen un derecho a participar en el gobierno de la universidad... Yo no le tengo miedo a la participación de los jóvenes en el gobierno de la Universidad ni en ninguna de las estructuras del país, porque creo que a la juventud del país tenemos que educarla en la responsabilidad y la responsabilidad no es nunca una situación así expeditiva, es una verdadera asunción de deberes y obligaciones. Hagamos que los jóvenes de este país tomen todos sus deberes y tengamos confianza en ellos... Como miembro del Consejo Directivo de una de las Facultades, los he visto actuar, algunas veces con pequeñeces y otras con grandeza... si la idea de la autonomía fundamenta la idea de idoneidad... la idoneidad comprende también a los estudiantes que son miembros de la comunidad universitaria “ AUNC, Actas de la Asam-blea...-, op. cit., fls 172 v y 173 r. (Palabras de Adelmo Montenegro).
} 
de reivindicar el triunfo de la libertad política, la democracia adquiría un sentido amplio y poner freno a las aspiraciones de los jóvenes se entendía como un ataque a la misma. Calificados intelectuales del siglo se habían encargado de denostar al voto calificado, "el eterno problema por el cual se pretende que la democracia debe circunscribirse al grupo más destacado intelectualmente". ${ }^{43} \mathrm{Y}$ su aplicación parecía ser lo que algunos pretendían para el caso de la universidad. Finalmente, en julio de 1958 , treinta y cinco asambleístas votaron el despacho de la mayoría consagrando la presencia de cuatro estudiantes en los órganos de gobierno de la universidad, lo cual constituyó el primer peldaño para futuras conquistas.

\section{CONCLUSIONES}

Estas medulosas discusiones, que demandaron largas jornadas a aquel grupo de intelectuales conformado por docentes, estudiantes y profesionales, nos sirven hoy para apreciar su pensamiento. Pero, en realidad, la cuestión estaba resuelta antes de empezar. Se había iniciado con el siglo, cuando el sector más ilustrado del país resaltó la capacidad de los jóvenes para ocupar cargos de responsabilidad creando una conciencia que, desde entonces, no pudo eludir ni siquiera el gobierno peronista reconocidamente autoritario; lo puso en marcha el gobierno de la Revolución Libertadora al dictar las primeras leyes al respecto, pensando suprimir para siempre los excesos del totalitarismo y queriendo "recompensar" a la juventud por su actitud durante las difíciles jomadas que culminaron en septiembre de 1955; lo puso en práctica aquel primer Consejo Universitario, que logró establecer una fórmula transaccional que preservara una ligera superioridad para el estamento docente. El resto dependería de la destreza que los estudiantes, en los años sucesivos, fuesen capaces de desarrollar para lograr sus fines. Muchos de aquellos profesores creyeron que estaban dando un importante paso hacia la superación de la crisis y el engrandecimiento de las universidades y, los jóvenes, supieron que habían ganado la primera e importante batalla para acceder al gobierno de las altas casas de estudio.

En un país que aún busca su identidad y donde existen marcadas tendencias imitativas, el proceso que hemos reseñado adquiere connotaciones significativas. Se trataba de conformar una universidad argentina, según un modelo argentino, que trabajara con sus hombres y con su realidad, para superar situaciones complejas. ${ }^{44} \mathrm{El}$ modelo no tuvo continuidad, pues la autonomía universitaria, proclamada orgullosamente al culminar los ' 50 , no perduró siempre. Los gobiernos militares recurrieron a la intervención de las altas casas de estudio y a

${ }^{43}$ AUNC, Actas de la Asamblea..., tomo I, op. cit., fl. 170 r. (Palabras de Jorge Orgaz)

${ }^{44}$ AUNC, Actas de la Asamblea..., tomo I, op. cit., fl. 171 v. (Palabras de Jorge Orgaz). 
la consecuente eliminación de los órganos colegiados de gobierno. Pero, desde 1983, ellas parecen haber ingresado en un período de estabilidad y en su estructura, el papel del estudiante en el gobierno tiende a ser decisivo. No obstante, también es cierto que han transcurrido 45 años desde 1958 y la crisis universitaria tiene hoy matices que parecen insuperables en el corto y mediano plazo, acrecentada por problemas tales como la falta de presupuesto y el excesivo número de alumnos para la infraestructura existente ${ }^{45}$. No obstante, se debe destacar que este proceso que hemos descrito fue original, buscando en la juventud aquello que los mayores no habían sabido conseguir. Puso en manos de jóvenes, sólo portadores de un título secundario, la responsabilidad compartida de dirigir la enorme estructura de las universidades nacionales, creadas para otorgar títulos de grado. Actualmente ellos cargan sobre sus hombros con ese compromiso y, aunque muchos no sean ni siquiera conscientes, son también responsables tanto de las frustraciones y fracasos que se viven hoy en las altas casas de estudio como, por supuesto, también de sus éxitos.

\section{FUENTES}

\section{ARCHIVO DE LA UNIVERSIDAD NACIONAL DE CÓRDOBA}

Actas del Honorable Consejo Universitario, Tomo I, (14 de junio al 15 de noviembre de 1956).

Actas del Honorable Consejo Superior, Tomo I (24 de enero al 25 de abril de 1958).

Actas de la Asamblea Universitaria, 1957-1958.

Anales de Legislación Argentina, 1947 y 1956, Tomos VII y XVI A, Buenos Aires, La Ley.

Jurisprudencia Argentina, Buenos Aires, años 1947, 1955 y 1956.

\section{SELECCIÓN BIBLIOGRÁFICA}

AARNIO AULIS, Ernesto Garzón Valdés y Jyrki Usitalo (comp.) La normatividad del derecho, Barcelona, Gedisa, 1997.

ÁLVAREZ DE TOMASSONE, Delia Teresita. UNIV ERSIDAD OBRERA NACIONAL - UNIVERSIDAD TECNOLÓGICA NACIONAL. La génesis de una Universidad (1948 - 1962), Editorial de la Universidad Tecnológica Nacional, 2006.

${ }^{45}$ Soy consciente que hoy muchos se niegan a reconocer que tal crisis exista, pero el demostrarlo amerita otra comunicación. 
BOBBIO, Norberto. (1990): Contribución a la teoría del derecho, Madrid, Editorial Debate.

BOBBIO, Norberto. (1995): MATTEUCCI, Nicola y Gianfranco Pasquino, Diccionario de Política, México, Siglo Veintiuno Editores (octava edición en español).

CIRIA, Alberto y SANGUINETI, Horacio. (1986): La Reforma Universitaria 1918 - 1983, tomo I, Centro Editor de América Latina, Biblioteca Política Argentina, Ediciones Especiales.

DEL MAZO, Gabriel (Compilador), La Reforma Universitaria, Tomo I: El Movimiento Argentino (1918 - 1940), La Plata, Edición del Centro de Estudiantes de Ingeniería, 1941.

KORN, Alejandro. (1986): "La Reforma Universitaria" en Alberto Ciria y Horacio Sanguineti, La Reforma Universitaria 1918 - 1983, tomo I, Centro Editor de América Latina, Biblioteca Política Argentina, Ediciones Especiales.

LA PORTA, Francisco. (1996): “Poder y derecho” en Ernesto Garzón Valdés y Francisco Laporta (Ed.) El derecho y la justicia, Madrid, Consejo Superior de Investigaciones Científicas, Boletín Oficial del Estado, Editorial Trotta.

MARIANI, Víctor. (1986): “Introducción al estudio del poder” en Héctor Roudil (Compilador), Estudios sobre la Sociedad y el Estado, Buenos Aires, Eudeba.

MILLS Wright, La élite del poder, México - Buenos Aires, Fondo de Cultura Económica, 1957.

Municipalidad de Córdoba, La Reforma Universitaria 1918 - 1988, Buenos Aires, Ministerio de Educación y Justicia de la Nación, Universidad Nacional de Córdoba y Fundación Friedrich Ebert, 1989.

PORTO AVELINO, José "Las Leyes, la Universidad y el País" en Todo es Historia, n 147, agosto de 1979, 24.

RIQUELME, Norma Dolores. (2002): "Los espacios de poder en la universidad argentina de mediados del siglo XX. El caso de Córdoba" en Actas do XII Congreso Internacional de Abila, volumen IV, editadas por Eugenio Dos Santos, Asociación de Historiadores Latinoamericanistas Europeos (AHILA) y Centro Leonardo Coimbra, Faculdade de Letras da Universidade do Porto. 
RIQUELME, Norma Dolores. (2003): “El debate acerca de los espacios de poder en la universidad argentina de mediados del siglo XX. El caso de Córdoba", en Primer Congreso Internacional sobre Historia de las Universidades en América y Europa, Córdoba.

ROUDI, Héctor. (1986):(Compilador), Estudios sobre la Sociedady el Estado, Buenos Aires, Eudeba.

SARLO, Beatriz. (2001): La batalla de las ideas (1943-1973), Biblioteca del Pensamiento Argentino, VII, Buenos Aires, Ariel.

VANOSSI, Reinaldo. (1989): "Balance de los setenta años de la Reforma Universitaria” en Municipalidad de Córdoba, La Reforma Universitaria 1918 - 1988, Buenos Aires, Ministerio de Educación y Justicia de la Nación, Universidad Nacional de Córdoba y Fundación Friedrich Ebert, 1989.

WEBER, Max. (1997): Economía y sociedad. Esbozo de sociología comprensiva, México, Fondo de Cultura Económica, (undécima reimpresión).

RIQUELME, Norma Dolores. (2008): “Conformación de los espacios de poder en el gobierno de la universidad nacional de córdoba (argentina) A mediados del siglo XX", en Revista Historia de la Educación Latinoamericana $\mathrm{N}^{\mathrm{0}}$ 11, Tunja, Universidad Pedagógica y Tecnológica de Colombia, RUDECOLOMBIA, pp. 167-188. 